of NAFLD is almost entirely the result of transition between absence to light NAFLD for subjects in the range 18-30 years old. A small effect of BMI was also seen in post-menopause women.

Conclusions The implications of this alarming and silent prevalence of NAFLD derived from the fact that this liver condition may progress to more severe liver disease.

\section{P2-230 OVERWEIGHT AND OBESITY: PREVALENCE AND THEIR ASSOCIATION WITH SOME SOCIAL AND LIFE-STYLE CHARACTERISTICS IN A RANDOM SAMPLE POPULATION- BASED STUDY IN SOUTHERN ITALY}

\author{
doi:10.1136/jech.2011.142976j.63
}

\begin{abstract}
${ }^{1} \mathrm{~A}$ Osella, ${ }^{2} \mathrm{M}$ del Pilar Díaz, ${ }^{1} \mathrm{R}$ Cozzolongo, ${ }^{1} \mathrm{G}$ Leandro, ${ }^{1} \mathrm{~S}$ Elba, ${ }^{1} \mathrm{~J}$ Petruzzi, ${ }^{1} \mathrm{G}$ Buongiorno, ${ }^{1} \mathrm{~V}$ Gianuzzi. IIRCCS Saverio de Bellis, Castellana Grotte, Bari, Italy; ${ }^{2}$ Escuela de Nutrición, Facultad de Ciencias Médicas, Universidad Nacional de Córdoba, Córdoba, Argentina
\end{abstract}

Introduction Obesity has become a threat to public health, as the epidemic is not confined to developed countries but is affecting many developing ones.

Objectives To estimate overweight and obesity prevalence and their association with some social characteristics in a population older than 18 years of age.

Methods The survey was conducted from July 2005 to January 2007 in Putignano, Bari, Apulia. A systematic random sample of 2301 subjects from the list of records maintained by general practitioners was drawn. Subjects were asked to complete a questionnaire regarding sociodemographic characteristics and life styles, underwent anthropometric measures and liver echography. A fasting blood sample was drawn. A quantile regression model was fitted to the data (conditional quantiles: 0.47 overweight and 0.83 obesity) with 500 bootstrapped replications.

Results Overall prevalence of overweight was 50.1\% (male 59.5\%, females $14.6 \%$ ) whereas obesity prevalence was $16.1 \%$ (male $18.0 \%$, females $14.6 \%$ ). Overweight and obesity steady raised with age. Overweight was associated positively with age at enrolment, being a male, number of household members, daily alcohol intake, married status and high socio-economic status. Obesity was associated positively with age at enrolment, being a male and daily alcohol intake.

Conclusion There is little doubt that socio-cultural and life-style factors operate to influence overweight and obesity but it is important to investigate the factors and their interaction on obesity as well as the pathway linking them. This approach may support the development and implementation of effective public health strategies.

\section{P2-231 STOMACH CANCER MORTALITY AMONG AGRICULTURAL WORKERS: RESULTS FROM A BRAZILIAN DEATH CERTIFICATE-BASED CASE-CONTROL STUDY}

\section{doi:10.1136/jech.2011.142976j.64}

${ }^{1} \mathrm{P}$ Boccolini, ${ }^{1} \mathrm{~J}$ Chrisman, ${ }^{1} \mathrm{U}$ Otero, ${ }^{*}{ }^{2} \mathrm{~S}$ Markowitz, ${ }^{3} \mathrm{~A}$ Meyer. ${ }^{1}$ National Cancer Institute, Rio de Janeiro, Brazil, ${ }^{2}$ Center for the Biology of Natural Systems, Queens College, City University of New York, USA; ${ }^{3}$ Federal University of Rio de Janeiro, Rio de Janeiro, Brazil

Introduction The use of pesticides in Brazil has been high. It is important to assess the risks related to the use of these substances. Some studies have reported an association between agricultural work and stomach cancer. This study aimed to estimate the risk of death from stomach cancer among agricultural workers in an intensive pesticide-use area in Brazil, 1996-2005.
Methods Cases were men and women aged $\geq 20$ years old that died of stomach cancer. For each case two age and sex matched controls were randomly selected, from all possible controls where the cause of death was not cancer or haematological disease. Crude and adjusted ORs were estimated to evaluate the magnitude of the risk. Results Agricultural workers living in the Serrana Region of Rio de Janeiro State were at higher risk of death from stomach cancer (OR: 1.41 (95\% CI 1.10 to 1.82)) compared to non-agricultural workers. Stratified analysis revealed that the risk was higher among younger (OR: 3.34 (1.58 to 7.08)) agricultural workers.

Conclusion These results suggest that agricultural workers living in the Serrana Region of the state of Rio de Janeiro were at statistically significant higher risk of death from stomach cancer compared to non-agricultural workers.

\section{P2-232 CARDIOVASCULAR DISEASE CONDITIONS: PREVALENCE, AWARENESS, TREATMENT AND CONTROL AMONG THE URBAN POOR IN NAIROBI}

doi:10.1136/jech.2011.142976j.65

S Oti, ${ }^{*}$ C Kyobutungi. African Population and Health Research Center, Nairobi, Kenya

Introduction The burden of Non-communicable diseases, including cardiovascular diseases (CVD), and their risk factors are rising in Sub Saharan Africa. In Kenya, prevalence of overweight and obesity among reproductive-age females almost doubled over a fifteen-year period $(13 \%-23 \%)$ between 1993 and 2008. This may be attributable to rapid urbanisation and unhealthy lifestyles. Little is known about prevalence and awareness levels of CVD in Sub Saharan Africa. This study describes burden and treatment patterns of common CVD conditions (diabetes and hypertension) among adults in two urban slum settlements in Nairobi, Kenya.

Methods We conducted a cross-sectional survey on a stratified random sample of 5190 adults aged 18 years and older in an area under the Nairobi Urban Health Demographic Surveillance System. Data collected included self- reported diagnosis and treatment history of hypertension and diabetes, blood pressure and glucose measurements

Results The prevalence of hypertension and diabetes was $12.3 \%$ and $4.3 \%$ respectively. Only one in five of those diagnosed with diabetes or hypertension was aware of their condition. Among those aware of having hypertension, less than one third were on treatment, and only $10 \%$ were controlled (blood pressure $<140 / 90 \mathrm{~mm} \mathrm{Hg}$ ). Among those who were aware of being diabetic, just over half were on treatment and one-fifth had Random Blood Sugar $<7.7 \mathrm{mmol} / \mathrm{dl}$. Conclusion The burden of common CVD among these poor communities is quite high. Worse, the level of awareness, treatment and control are dismally low. Interventions to increase awareness for example, through routine adult screening are needed to avert an impending CVD epidemic.

\section{P2-233 ASSOCIATION BETWEEN SLEEP DURATION AND BLOOD PRESSURE IN ADOLESCENTS}

doi:10.1136/jech.2011.142976j.66

1,2 Paciência, * 2,3 J Araújo, ${ }^{1,2}$ E Ramos. ${ }^{1}$ Institute of Public Health, University of Porto, Porto, Portugal; ${ }^{2}$ Department of Hygiene and Epidemiology, University of Porto Medical School, Porto, Portugal; ${ }^{3}$ Cardiovascular Research \& Development Unit, University of Porto Medical School, Porto, Portugal

Background In adults, sleep has showed an important role on health namely in cardiovascular risk factors. The purpose of this crosssectional study was to evaluate the association between sleep duration and blood pressure, at 13 years of age. 